\title{
An Efficient Frequency Domain State-Space Identification Algorithm
}

\author{
Tomas McKelvey and Hüseyin Akçay \\ Department of Electrical Engineering \\ Linköping University \\ S-581 83 Linköping, Sweden \\ e-mail: Tomas.McKelvey@isy.liu.se \\ Fax: +4613282622
}

February 23, 1994

\begin{abstract}
33rd CDC
Abstract

In this paper we present a novel non-iterative algorithm for identifying linear time-invariant discrete time state-space models from frequency response data. We show that the algorithm recover the true system of order $n$ if $n+2$ noise-free frequency response measurements are given at uniformly spaced frequencies. The algorithm is demonstrated to be related to the recent timedomain subspace identification algorithms formulated in the frequency domain. The algorithm is applied to real frequency data, originating from a flexible mechanical structure, with promising results. In a companion paper robustness and stochastic analysis is performed.
\end{abstract}

Keywords: system identification, state-space methods, frequency response.

\section{Introduction}

Identification of large scale multi-input multi-output (MIMO) systems of high orders is still considered a challenge. This type of systems are encountered in the mechanical engineering area of modal analysis and in the control of flexible structures. In most cases it is desired to obtain a single model in a minimal realization which makes state-space models the best choice which also facilitates controller design since most modern multivariable design techniques requires a state-space model of the system.

If time domain measurements are available a vast number of different algorithms exist in the literature. The algorithms can be divided in two groups; iterative methods and non-iterative methods. Among the iterative we find the prediction-error methods [14] and among the noniterative we find the more recent sub-space based algorithms [16, 20, 13]. The advantage of the non-iterative methods is the absence of a nonlinear parametric optimization. Hence the methods always produce a result and never reach local minima. The disadvantages is the poor knowledge of the performance of the methods with finite data records. 
In practice information about a system is often obtained as frequency response samples of the transfer function at some discrete frequencies. These are usually obtained from high performing sophisticated data analyzers and data acquisition equipment and are of high quality.

The problem of fitting a real-rational model to a given frequency response has been addressed by many authors. The most natural way is to model the system as a fraction of two polynomials $(a, b)$ with real coefficients and solve the problem

$$
\hat{a}\left(e^{i \omega}\right), \hat{b}\left(e^{i \omega}\right)=\arg \min _{a, b} \sum_{k=1}^{N}\left|\frac{b\left(e^{i \omega_{k}}\right)}{a\left(e^{i \omega_{k}}\right)}-G_{k}\right|^{2}
$$

where $G_{k}$ are the transfer function measurements at frequencies $\omega_{k}$. However the solution to this problem formulation involves a nonlinear parametric optimization just as the prediction error methods in the time domain.

In early results [10] a linear least-squares formulation was suggested an further refined in [18] (SK-iterations) by solving a sequence of linear least-square problems. However these methods do not always converge to the minimum of (1) as pointed out by [21]. A second drawback is the parameterization of the model. The poles and zeros of the system become very sensitive to perturbations in the coefficients of the polynomials if the system order is high. This deficiency can be reduced by introducing other parameterizations ,e.g. orthogonal Chebyshev polynomials [3, 1] or Zero-Pole-Gain form or the related RPM-parameterization [17].

Some more recent methods are non-iterative and based on Inverse Discrete Fourier transform techniques to obtain estimates of the impulse response an then apply the realization algorithms by Ho and Kalman [6], Kung [9] or the ERA-algorithm [7]. These realization algorithms find a minimal state space realization given the first part of the impulse response (the Markov parameters). The fundamental problem with this approach is that the estimated impulse response always will be perturbed since, in reality only a finite number of frequency response measurements are available. In [8] estimates of the impulse response are constructed by recursive scheme. This approach is however exact only if the impulse response dies out completely within the number of frequency points given. For lightly damped systems this approach yields very poor estimates.

In [2] Bayard suggests to first fit a high order rational model using the SK-iterations and then calculate the impulse response (the Markov parameters) using the high order model. Model reduction to a low order state-space model is then applied by utilizing the realization algorithm in $[7]$.

A new frequency domain approach has been proposed by Liu and co workers [12] which is a frequency domain counterpart of the time domain subspace methods in [16] [13]. This approach does not require the data to be uniformly spaced in frequencies and also offers some frequency weighting capabilities.

This paper introduce a novel frequency domain state-space identification algorithm using the frequency response of a system. The major features of this new approach are: A system of order $n$ is exactly recovered using $n+2$ frequency response samples if the data is noise-free. The algorithm is non-iterative and involves 3 key steps. First an IDFT is performed to obtain (distorted) impulse response estimates. Secondly the estimates are used in a realization step, by a singular value decomposition (SVD), to obtain the $A$ and $C$ matrices. Even though the impulse response estimates are distorted, these estimates of $A$, and $C$ are correct (in the noise-free case). In the third step $B$ and $D$ are estimated by an ordinary least-squares method. It will also be shown that the subspace approach of Liu et.al. [12] is related this method if the frequencies are uniformly spaced. As an 
illustration we employ the algorithm on real data originating ${ }^{1}$ from a frequency response experiment on a flexible structure from the Jet Propulsion Laboratory, Pasadena, California. These data has also been used in [5]. The example clearly indicate that the subspace methods are competitive compared with classical iterative least-square methods. In a companion paper the proposed algorithm is successfully analyzed with respect to robustness to norm bounded perturbations and consistency when the measurements are corrupted with additive noise.

\section{Problem formulation}

We will assume that the true system $G$ is a stable multivariable linear time-invariant discrete time system with input/output properties characterized by the impulse response coefficients $g_{k}$ through the equation

$$
y(t)=\sum_{k=0}^{\infty} g_{k} u(t-k)
$$

where $y(t) \in \mathbb{R}^{p}, u(t) \in \mathbb{R}^{m}$ and $g_{k} \in \mathbb{R}^{p \times m}$. If the system is of finite order $n$ it can be described by a state-space model

$$
\begin{aligned}
x(t+1) & =A x(t)+B u(t) \\
y(t) & =C x(t)+D u(t)
\end{aligned},
$$

where $y(t) \in \mathbb{R}^{p}, u(t) \in \mathbb{R}^{m}$, and $x(t) \in \mathbb{R}^{n}$. The state-space model (3) is a special case of (2) with

$$
g_{k}=\left\{\begin{array}{ll}
D, & k=0 \\
C A^{k-1} B, & k>0
\end{array} .\right.
$$

The frequency response of (2) is

$$
G\left(e^{j \omega}\right)=\sum_{k=0}^{\infty} g_{k} e^{-j \omega k}
$$

which for the state-space model (3) can be written as

$$
G\left(e^{j \omega}\right)=C\left(e^{j \omega} I-A\right)^{-1} B+D .
$$

The problem formulation is then; Given a finite number $M+1$, possibly noisy, samples

$$
G_{k}=G\left(e^{j \omega_{k}}\right)+e_{k}, \omega_{k} \in[0, \pi]
$$

of the frequency response of the system find a finite dimensional state-space system (3) of order $n$, denoted by $\hat{G}$, such that the true system and the identified model are "close", where the closeness is quantified by the following distance between the true and estimated transfer functions

$$
\|G-\hat{G}\|_{\infty} \triangleq \sup _{\omega} \bar{\sigma}\left(G\left(e^{j \omega}\right)-\hat{G}\left(e^{j \omega}\right)\right) .
$$

Here $\bar{\sigma}(A)$ denote the largest singular value of the $p \times m$ matrix $A$. Furthermore, denote by $\sigma_{i}(A)$ the $i$ th singular value, where the ordering is given by $\sigma_{1}(A) \geq \sigma_{2}(A) \geq \ldots \geq \sigma_{r}(A) \geq 0$ with $r=\min (p, m)$.

\footnotetext{
${ }^{1}$ The authors have received the data from P. Khargonekar but have not yet managed to reach D. Bayard for an official approval to use the data.
} 


\section{The algorithm}

If the impulse response coefficients (4) are given, well-known realization algorithms can be used to obtain state-space realizations, see e.g. [6] and [9]. The algorithm to be presented is closely related to these results but does not require the true impulse response coefficients to be known.

Assume that frequency response data $G\left(e^{j \omega_{k}}\right)$ on a set of uniformly spaced frequencies, $\omega_{k}=$ $\frac{\pi k}{M}, k=0, \ldots, M$ are given. Since $G$ is a real transfer function, frequency response data on $[0, \pi]$ can be extended to $[0,2 \pi]$ as follows

$$
G\left(e^{j \pi(1+k / M)}\right)=G^{*}\left(e^{j \pi(M-k) / M}\right), \quad k=1, \ldots, M-1
$$

where $(\cdot)^{*}$ denotes complex conjugate. Construct the impulse response block Hankel matrix

$$
\hat{H}_{q r}=\left[\begin{array}{cccc}
\hat{g}_{1} & \hat{g}_{2} & \ldots & \hat{g}_{r} \\
\hat{g}_{2} & \hat{g}_{3} & \ldots & \hat{g}_{r+1} \\
\vdots & \vdots & \ddots & \vdots \\
\hat{g}_{q} & \hat{g}_{q+1} & \ldots & \hat{g}_{q+r-1}
\end{array}\right]
$$

with number of block rows $q>n$ and block columns $r \geq n$ and where the first $q+r-1$ impulse response coefficients of $G$ are estimated from the $2 M$-point Inverse Discrete Fourier Transform (IDFT) according to

$$
\hat{g}_{i}=\frac{1}{2 M} \sum_{k=0}^{2 M-1} G\left(e^{j 2 \pi k / 2 M}\right) e^{j 2 \pi i k / 2 M}, i=0, \ldots, q+r-1 .
$$

The size of $\hat{H}_{q r}$ is limited by $q+r \leq M$.

Compute the singular value decomposition of $\hat{H}_{q r}$

$$
\hat{H}_{q r}=\left[\begin{array}{ll}
\hat{U}_{1} & \hat{U}_{2}
\end{array}\right]\left[\begin{array}{cc}
\hat{\Sigma}_{1} & 0 \\
0 & \hat{\Sigma}_{2}
\end{array}\right]\left[\begin{array}{l}
\hat{V}_{1}^{T} \\
\hat{V}_{2}^{T}
\end{array}\right]
$$

where $\hat{\Sigma}_{1}$ contains the $n$ principal singular values.

The system matrices are then estimated as

$$
\begin{gathered}
\hat{A}=\left(\left[\begin{array}{cc}
I_{(q-1) p} & 0_{(q-1) p \times p}
\end{array}\right] \hat{U}_{1} \hat{\Sigma}_{1}^{1 / 2}\right)^{\dagger}\left(\left[\begin{array}{ll}
0_{(q-1) p \times p} & I_{(q-1) p}
\end{array}\right] \hat{U}_{1} \hat{\Sigma}_{1}^{1 / 2}\right) \\
\hat{C}=\left[\begin{array}{ll}
I_{p} & 0_{p \times(q-1)}
\end{array}\right] \hat{U}_{1} \hat{\Sigma}_{1}^{1 / 2}, \\
\hat{B}, \hat{D}=\arg \min _{\hat{B}, \hat{D}} \sum_{i=0}^{M}\left|G\left(e^{j 2 \pi i / 2 M-1}\right)-\hat{G}\left(e^{j 2 \pi i / 2 M-1}\right)\right|^{2}
\end{gathered}
$$

where

$$
\hat{G}(z)=\hat{D}+\hat{C}(z I-\hat{A})^{-1} \hat{B}
$$

and $I_{i}$ denotes the $i \times i$ identity matrix and $0_{i \times j}$ denotes the $i \times j$ zero matrix and $A^{\dagger}=\left(A^{T} A\right)^{-1} A^{T}$ denotes the Moore-Penrose pseudo-inverse of the full-rank matrix $A$. Notice that $B$ and $D$ appear linear in (16) and thus (15) can be solved by a linear least-squares solution. 
From (11), notice that

$$
\lim _{M \rightarrow \infty} \hat{g}_{k}=\int_{0}^{2 \pi} G\left(e^{j 2 \pi \theta}\right) e^{j 2 \pi k \theta} d \theta=g_{k}, k=0, \ldots, q+r-1,
$$

where $g_{k}$ is the $k$ th impulse response coefficient of $G$. Let $H_{q r}$ and $\Sigma_{i}, U_{i}, V_{i}, i=1,2$ denote the limits of the matrices in (12-16) as $M$ tends to infinity. The resulting algorithm is then known as Kung's algorithm [9], in which $\hat{B}$ is calculated from

$$
\hat{B}=\Sigma_{1}^{1 / 2} V_{1}^{T}\left[\begin{array}{c}
I_{m} \\
0_{m \times(r-1)}
\end{array}\right] .
$$

The realization given by (12-16) in Kung's algorithm [9] is balanced in the sense that the $q$-block row observability matrix

$$
\mathcal{O}_{q}=\left[\begin{array}{c}
C \\
C A \\
\vdots \\
C A^{q-1}
\end{array}\right]
$$

and the $r$-block column controllability matrix

$$
\mathcal{C}_{r}=\left[\begin{array}{llll}
B & A B & \ldots & A^{r-1} B
\end{array}\right]
$$

satisfies

$$
\mathcal{O}_{q}^{T} \mathcal{O}_{q}=\mathcal{C}_{r} \mathcal{C}_{r}^{T}=\Sigma_{1}
$$

A true balanced realization will thus be obtained only if both $q$ and $r$ tend to infinity. In this limiting case $\Sigma_{1}$ equals the Hankel singular values of the system $G$, see [4]. For finite $q$ and $r$ the Hankel singular values of $G$ are underestimated by $\Sigma_{1}$. Although the singular values $\Sigma_{1}$ do not play any role (except for a selection of a base for the state-space variables) in the construction of a state-space model for $G$, they will be essential in the selection of a model order in the presence of unmodelled dynamics and noise. (Notice that $A$ and $C$ can be calculated from (12-14) letting $\left.\Sigma_{1}=I_{n}\right)$. As $q$ and $r$ increase, the singular values of $H_{q r}$ also increase in the absence of noise. However, small singular values of $H_{q r}$ and corresponding left and right singular vectors will be less reliable in the presence of noise. Therefore $q$ and $r$ should be chosen sufficiently large to obtain $\Sigma_{1}$ as large as possible and the model order should be kept as low as possible. We will formalize these observations in a succeeding section.

The spectral radius of a square $n \times n$ matrix is defined as

$$
\rho(A)=\max \{|\lambda|: \lambda \in \lambda(A)\}
$$

where $\lambda(A)$ are the eigenvalues of $A$.

The usefulness of the above presented algorithm in the case of finite $M$ follows from the following key theorem.

Theorem 3.1 Let $G$ be an nth order system represented by (3). Assume that $\rho=\rho(A)<1$. Then $n+2$ noiseless equidistant frequency response measurements of $G$ on $[0, \pi]$ are sufficient to identify $G$ by the above algorithm (9-16) and

$$
\hat{\Sigma}_{1} \geq\left(1+\rho^{2 M}\right)^{-1} \Sigma_{1}
$$


Proof: Since $G$ is a stable transfer function, it can be represented by the following Taylor series

$$
G(z)=D+C(z I-A)^{-1} B=D+\sum_{k=1}^{\infty} C A^{k-1} B z^{-k}=\sum_{k=0}^{\infty} g_{k} z^{-k}, \rho<|z| .
$$

Notice that $\hat{g}_{k}$ can be written as

$$
\hat{g}_{k}=\sum_{i=0}^{\infty} g(k+2 i M)=C A^{k-1}\left(\sum_{i=0}^{\infty} A^{2 i M}\right) B=C A^{k-1}\left(I-A^{2 M}\right)^{-1} B=C A^{k-1} \tilde{B}
$$

and therefore $\hat{H}_{q r}$ can be factored as

$$
\hat{H}_{q r}=\mathcal{O}_{q} \tilde{\mathcal{C}}_{r}=\mathcal{O}_{q}\left[\begin{array}{llll}
\tilde{B} & A \tilde{B} & \ldots & A^{r-1} \tilde{B}
\end{array}\right] .
$$

Hence, if $r \geq n$ and $q>n$, then $\hat{\Sigma}_{2}=0$ and the column range spaces of $H_{q r}$ and $\hat{H}_{q r}$ will be equal. Thus $\hat{A}, \hat{C}, \hat{G}$ are related to $A, C$ and $G$ by a square nonsingular matrix $S$ as

$$
\hat{A}=S^{-1} A S ; \quad \hat{C}=C S ; \quad \hat{G}=\hat{D}+C(z I-A)^{-1} S \hat{B} .
$$

Since $(15)$ has a unique solution, we get $\hat{B}=S^{-1} B, \hat{D}=D$. Then $(A, B, C, D)$ and $(\hat{A}, \hat{B}, \hat{C}, \hat{D})$ are similar. Letting $q=n+1, r=n, M=n+2$, we satisfy the condition $q+r<2 M$.

For the second part: notice that $\hat{H}_{q r} \hat{H}_{q r}^{T}-\left(1+\rho^{2 M}\right)^{-2} H_{q r} H_{q r}^{T}$ is positive semidefinite if $\tilde{\mathcal{C}}_{r} \tilde{\mathcal{C}}_{r}^{T}-$ $\left(1+\rho^{2 M}\right)^{-2} \mathcal{C}_{r} \mathcal{C}_{r}^{T}$ is positive semidefinite which is true.

In the algorithm given by (12-15), the singular values of $\hat{H}_{q r}$ only influence the basis chosen for the state-space variables and not the transfer function $G$. Since in a realistic problem, $\hat{\Sigma}_{2}$ is never zero due to noise and unmodelled dynamics, a clear separation between the singular values of $\hat{\Sigma}_{1}$ and $\hat{\Sigma}_{2}$ must be obtained in order to choose a model order unambiguously. Theorem 3.1 gives a bound on the distortion of the smallest singular value due to aliasing caused by the finite $2 M$-point inverse DFT.

\subsection{Model order selection by cross validation}

Model validation is what distinguishes system identification from curve fitting. In system identification it is assumed that the measured data is generated by a finite dimensional system with some additive noise. To find a good model order selection criteria is then an interesting problem since increasing the model order always decrease the estimation error evaluated on the estimation data (unless numerical problems occur or, if iterative methods are used, local minima are reached). If we increase the model order above the true order the model will start to fit to the noise. In time domain identification a common solution to this is cross validation, see [19]; divide the data set in two parts and use one part for the identification and the other part for model validation. If the model order of the estimated model now increase over the correct model order we will see no decrease in the model quality using the independent validation data set. If the true system however is of infinite dimension cross validation techniques will not give the same guidance since an increased model order always will approximate the underlying infinite dimensional system better since the amount of unmodelled dynamics will decrease. However utilizing the cross validation step will tell us if the data is noisy measurements of a finite dimensional system or is from a noise free infinite dimensional system. 
In the frequency domain, cross validation is easily performed by dividing the frequency measurements in two disjoint sets; estimation data and validation data. The most natural division is to take every other frequency point as the estimation data and the rest as validation data. This division also preserves the uniformly spaced frequencies. A model is then estimated using the estimation data only. The quality of the model is assessed by comparing the estimated transfer function with the validation data set and a proper model order can then be inferred.

\section{Frequency domain subspace identification}

In [12] a sub-space based frequency domain identification algorithm is described. The algorithm is a frequency domain counterpart to the known algorithms in the time domain $[16,20,13]$ and others. We will briefly describe the approach by [12] and show a relation with the presented algorithm (9-16). To simplify notation we restrict ourself to single-input single-output systems. However with more notational effort the same derivation holds for multivariable systems.

Consider the state-space system (3). By concatenating outputs and inputs as

$$
y_{q}(k)=\left[\begin{array}{c}
y(k) \\
y(k+1) \\
\vdots \\
y(k+q-1)
\end{array}\right] ; \quad u_{q}(k)=\left[\begin{array}{c}
u(k) \\
u(k+1) \\
\vdots \\
u(k+q-1)
\end{array}\right] \text {, }
$$

and introducing the Toeplitz matrix

$$
\Gamma_{q}=\left[\begin{array}{cccc}
D & 0 & \ldots & 0 \\
C B & D & \ldots & 0 \\
\vdots & \vdots & \ddots & \vdots \\
C A^{q-2} B & C A^{q-3} B & \ldots & D
\end{array}\right]
$$

we obtain the relation

$$
y_{q}(k)=\mathcal{O}_{q} x(k)+\Gamma_{q} u_{q}(k)
$$

where $\mathcal{O}_{q}$ is the extended observability matrix (19). On taking the Fourier transform of both sides of (30), we obtain

$$
W(\omega) Y(\omega)=\mathcal{O}_{q} X(\omega)+\Gamma_{q} W(\omega) U(\omega)
$$

with

$$
W(\omega)=\left[\begin{array}{lllll}
1 & e^{j \omega} & e^{j 2 \omega} & \cdots & e^{j \omega(q-1)}
\end{array}\right]^{T} .
$$

Assume that $Y(\omega)$ and $U(\omega)$ are given at a discrete set of frequencies $\omega_{k} \in[0, \pi], k=1, \ldots, M$ and use these samples to define the following matrices;

$$
W_{q, M}=\left[\begin{array}{llll}
W\left(\omega_{1}\right) & W\left(\omega_{2}\right) & \cdots & W\left(\omega_{M}\right)
\end{array}\right]
$$




$$
\begin{aligned}
& Y_{q, M}=W_{q, M}\left[\begin{array}{cccc}
Y\left(\omega_{1}\right) & 0 & \cdots & 0 \\
0 & Y\left(\omega_{2}\right) & \cdots & \vdots \\
\vdots & \vdots & \ddots & \vdots \\
0 & \cdots & \cdots & Y\left(\omega_{M}\right)
\end{array}\right] \\
& U_{q, M}=W_{q, M}\left[\begin{array}{cccc}
U\left(\omega_{1}\right) & 0 & \cdots & 0 \\
0 & U\left(\omega_{2}\right) & \cdots & \vdots \\
\vdots & \vdots & \ddots & \vdots \\
0 & \cdots & \cdots & U\left(\omega_{M}\right)
\end{array}\right] \\
& X_{M}=\left[\begin{array}{llll}
X\left(\omega_{1}\right) & X\left(\omega_{2}\right) & \cdots & X\left(\omega_{M}\right)
\end{array}\right]
\end{aligned}
$$

Notice that $W_{q, M}$ is a Vandermonde matrix and thus always have full rank if the frequencies are distinct. With the matrices above and (30) we arrive at the complex matrix equation

$$
Y_{q, M}=\mathcal{O}_{q} X_{M}+\Gamma_{q} U_{q, M}
$$

which is the frequency domain counterpart of the time domain equation in [16].

\subsection{Observability Range Space Extraction in Frequency Domain}

There are several methods to extract the observability range space from (37). In (37), the real matrices $\mathcal{O}_{q}, \Gamma_{q}$ and the complex matrix $X_{M}$ are unknown. We begin with the construction of the following three matrices

$$
\mathcal{U}=\left[U_{q, M}, U_{q, M}^{*}\right], \quad \mathcal{X}=\left[X_{M}, X_{M}^{*}\right], \quad \mathcal{Y}=\left[Y_{q, M}, Y_{q, M}^{*}\right] .
$$

Then, from (37), we obtain

$$
\mathcal{Y}=\mathcal{O}_{q} \mathcal{X}+\Gamma_{q} \mathcal{U}
$$

Following the time domain work in [16] we form the following matrix

$$
\mathcal{U}^{\perp}=I-\mathcal{U}^{H}\left(\mathcal{U U}^{H}\right)^{-1} \mathcal{U}
$$

assuming that $\mathcal{U}$ has full rank and multiply (39) from right by $\mathcal{U}^{\perp}$ to obtain

$$
\mathcal{Y} \mathcal{U}^{\perp}=\mathcal{O}_{q} \mathcal{X} \mathcal{U}^{\perp}
$$

Notice that $\operatorname{rank}\left(\mathcal{U}^{\perp}\right)=2 M-q m$ since $\mathcal{U}^{\perp}$ projects onto the subspace of $\mathbb{R}^{2 M}$ that is orthogonal to the range space of $\mathcal{U}^{H}$. Hence, multiplication of (40) from right by $\mathcal{U}^{H}$ yields

$$
\mathcal{U}^{\perp} \mathcal{U}^{H}=\mathcal{U}^{H}-\mathcal{U}^{H}\left(\mathcal{U} \mathcal{U}^{H}\right)^{-1} \mathcal{U} \mathcal{U}^{H}=\mathcal{U}^{H}\left(I-\left(\mathcal{U} \mathcal{U}^{H}\right)^{-1} \mathcal{U} \mathcal{U}^{H}\right)=0
$$

The relation (42) is used in [12] to arrive at

$$
\mathcal{Y} \mathcal{Y}^{H}-\mathcal{Y} \mathcal{U}^{H}\left(\mathcal{U} \mathcal{U}^{H}\right)^{-1}\left(\mathcal{Y} \mathcal{U}^{H}\right)^{H}=\mathcal{O}_{q}\left(\mathcal{X} \mathcal{X}^{H}-\mathcal{X} \mathcal{U}^{H}\left(\mathcal{H} \mathcal{U}^{H}\right)^{-1}\left(\mathcal{X} \mathcal{U}^{H}\right)^{H}\right) \mathcal{O}_{q}^{T}
$$

which also can be written as

$$
\mathcal{Y} \mathcal{U}^{\perp} \mathcal{Y}^{H}=\mathcal{O}_{q} \mathcal{X} \mathcal{U}^{\perp} \mathcal{X}^{H} \mathcal{O}_{q}^{T}
$$


since $\mathcal{U}^{\perp}\left(\mathcal{U}^{\perp}\right)^{H}=\mathcal{U}^{\perp}$. The imaginary parts of the above equation are zero. The observability range space is then estimated by performing a SVD on $\mathcal{Y} \mathcal{U}^{\perp} \mathcal{Y}^{H}$ and we can then proceed as (13-16). If frequencies are close and/or $q$ is large $\mathcal{U} \mathcal{U}^{H}$ might be close to singular and [12] propose to use the pseudo-inverse with a proper threshold for zero when forming $\left(\mathcal{U U}^{H}\right)^{-1}$ in (43). An even better way of forming $\mathcal{U}^{\perp}$ without forming the ill-conditioned $\mathcal{U U}^{H}$ is by using the SVD;

$$
E \Sigma V^{H}=\mathcal{U}
$$

with $E \in \mathbb{C}^{q \times q}, V \in \mathbb{C}^{2 M \times q}$ and the singular values in the diagonal matrix $\Sigma \in \mathbb{R}^{q \times q}$. Using the properties of the SVD we easily obtain

$$
\mathcal{U}^{\perp}=I_{2 M}-V V^{H} .
$$

Now assume that $U\left(\omega_{k}\right)=1, \forall k$ and hence $Y\left(\omega_{k}\right)=G_{k}$, the frequency response of the system. Extend the frequencies as (9). Given frequency response data construct the following matrix

$$
G_{q, 2 M}=\left[\begin{array}{cccc}
G\left(e^{j \omega_{0}}\right) & G\left(e^{j \omega_{1}}\right) & \cdots & G\left(e^{j \omega_{2 M-1}}\right) \\
e^{j \omega_{0}} G\left(e^{j \omega_{0}}\right) & e^{j \omega_{1}} G\left(e^{j \omega_{1}}\right) & \cdots & \vdots \\
\vdots & \vdots & \ddots & \vdots \\
e^{j \omega_{0}(q-1)} G\left(e^{j \omega_{0}}\right) & e^{j \omega_{1}(q-1)} G\left(e^{j \omega_{1}}\right) & \cdots & e^{j \omega_{2 M-1}(q-1)} G\left(e^{j \omega_{2 M-1}}\right)
\end{array}\right]
$$

This matrix is equal to $\mathcal{Y}$ except for a permutation of the columns. Using the notation $X_{q, 2 M}$ for the sampled state frequency response matrix, we can derive the following equation as a special case of $(37)$

$$
G_{q, 2 M}=\mathcal{O}_{q} X_{q, 2 M}+\Gamma_{q} W_{q, 2 M}
$$

Since $W_{q, 2 M}$ has full rank and $r<M, W_{q, 2 M}$ has a right annihilator $W_{q, 2 M}^{\perp}$ and multiplying (48) from right by $W_{q, 2 M}^{\perp}$, we get

$$
G_{q, 2 M} W_{q, 2 M}^{\perp}=\mathcal{O}_{q} X_{q, 2 M} W_{q, 2 M}^{\perp}
$$

Let us assume that equidistant frequency response data $G\left(e^{j \pi k / M}\right), k=0, \ldots, M$ are available.

Then $W_{q, 2 M}$ is annihilated by the following matrix

$$
W_{q, r}^{\perp}=\frac{1}{2 M}\left[\begin{array}{cccc}
1 & 1 & \cdots & 1 \\
e^{j 2 \pi / 2 M} & e^{j 4 \pi / 2 M} & \cdots & e^{j 2 \pi r / 2 M} \\
\vdots & \vdots & \vdots & \vdots \\
e^{j 2 \pi(2 M-1) / 2 M} & e^{j 4 \pi(2 M-1) / 2 M} & \cdots & e^{j 2 \pi r(2 M-1) / 2 M}
\end{array}\right] .
$$

Furthermore, the $\left(i_{1}, i_{2}\right)$-th entry of $G_{q, 2 M} W_{q, r}^{\perp}$; is calculated as

$$
\begin{aligned}
G_{q, 2 M} W_{q, r}^{\perp}\left(i_{1}, i_{2}\right) & =\frac{1}{2 M} \sum_{k=0}^{2 M-1} G\left(e^{j 2 \pi k / 2 M}\right) e^{j 2 \pi k\left(i_{2}+i_{1}-1\right) / 2 M} \\
& =\hat{g}^{i}\left(i_{2}+i_{1}-1\right) ; i_{1}=1 \ldots q, i_{2}=1 \ldots r
\end{aligned}
$$

which is the $2 M$-point DFT of the frequency response data. Hence $G_{q, 2 M} W_{q, r}^{\perp}=\hat{H}_{q r}$ from (10) and thus the method of [12] and the algorithm (9-16) are related. The major difference from the the algorithm in [12] is the use of a low rank annihilator $W_{q, 2 M}^{\perp}$ instead of the full rank annihilator (40). This difference turns out to be significant in the stochastic analysis in [15]. 


\section{An example}

To illustrate the performance of the presented algorithm we will use a real data set obtained from the Jet Propulsion Laboratory, Pasadena, California. The data origin from a frequency response experiment on a flexible structure. As a comparison we will also use the nonlinear least-squares algorithm invfreqz in Matlab Signal Processing Toolbox, see [11], on the same data. invfreqz is a two step method: First a rational model is fitted using a linear least-square method, see [10]. In the second step an iterative nonlinear optimization is performed to minimize the sum of the squared identification errors. Since an optimization is involved local minima may be reached during the second step.

The same data set has also been used in [5] and we will below relate their results to ours.

The JPL-data consists of a total of $M=512$ complex frequency samples $G_{k}, k=1 \ldots 512$ in the frequency range $\omega_{k}^{c} \in[1.23,628] \mathrm{rad} / \mathrm{s}$ uniformly spaced and origin from a flexible structure and hence has several lightly damped modes. Our aim is to construct a discrete time model matching the given frequency response. For the discrete time models we map the continuous frequencies to discrete frequency points according to

$$
\omega_{k}=\frac{\pi \omega_{k}^{c}}{\omega_{M}^{c}}
$$

This is the equivalent of zero order hold sampling of a continuous time system choosing $\omega_{N}^{c}$ as the Nyquist frequency.

\subsection{Model quality assessment}

In order to compare different estimated models we use two indicators based on the experimental data:

- The largest distance between estimated model and measured data

$$
\|G-\hat{G}\|_{\infty e} \triangleq \max _{k}\left|G_{k}-\hat{G}\left(e^{i \omega_{k}}\right)\right| .
$$

- The $L_{2}$ norm of the error

$$
\|G-\hat{G}\|_{2 e} \triangleq \sqrt{\frac{1}{M} \sum_{k=1}^{M}\left|G_{k}-\hat{G}\left(e^{i \omega_{k}}\right)\right|^{2}} .
$$

where the subscript $e$ indicates that the measures are only estimates of the true norms.

\subsection{Results}

In all estimations the number of block rows $q$ and columns $r$ of (10) were chosen to be $q=r=512$ to obtain a maximal size Hankel matrix. Figure 1 depicts the magnitude of the measured frequency data together with an estimated model of order 24 using algorithm (9-16). The estimated model accurately picks up all the eleven dominant peaks. Increasing model order further improves the fit. Figure 2 shows the results of estimation of models of order $n=24-62$ using the algorithm presented in this paper and the nonlinear least-squares method (invfreqz). In the figure the $\|G-\hat{G}\|_{\infty e}$ error is given and the $\|G-\hat{G}\|_{2 e}$ error have the same characteristics. For model order 24 our algorithm 


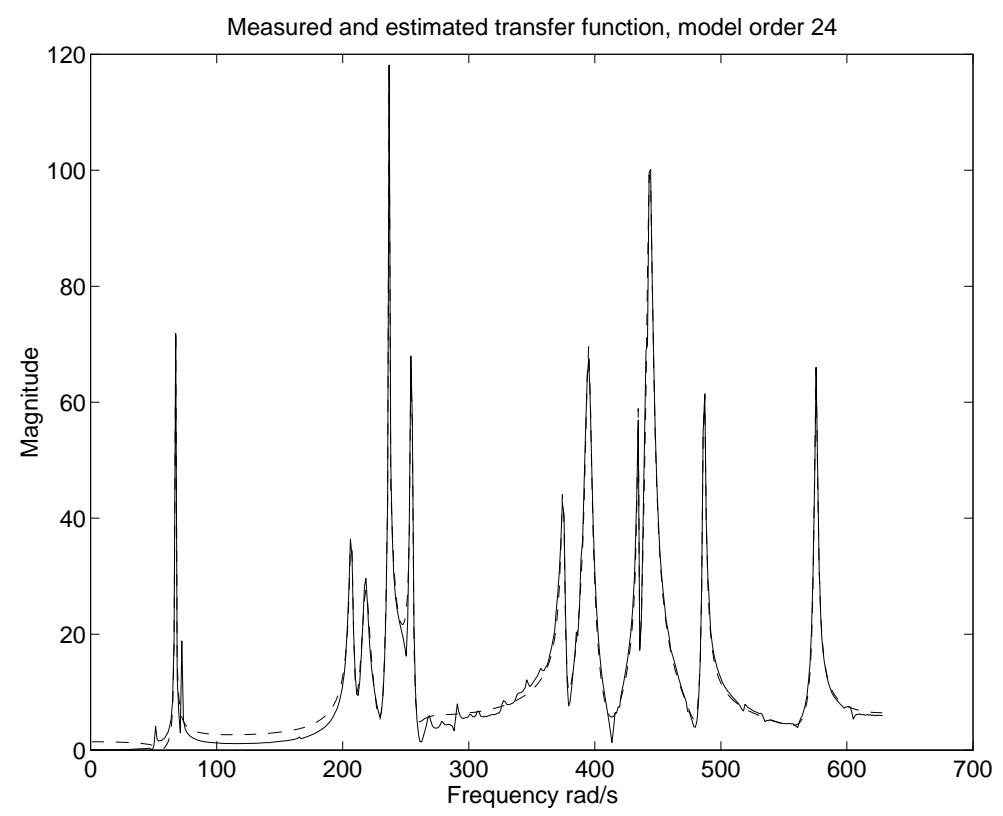

Figure 1: Magnitude of measured transfer function (solid line) and estimated model of order 24 using algorithm (9-16) (dashed line)

produces $\|G-\hat{G}\|_{\infty e}=13.2$ and for model order 42 we obtained $\|G-\hat{G}\|_{\infty e}=2.3$. It is quite clear that, for this data, the algorithm outperform the least-squares method for all model orders. This data set has also been used by $\mathrm{Gu}$ and Khargonekar in the paper [5]. They present a model of order 24 with $\|G-\hat{G}\|_{\infty e}=13$ which was obtained by SK iterations, see [18]. By introducing a second step and increasing model order to 42 they reduced the error to $\|G-\hat{G}\|_{\infty e}=6.1$. Comparing these results with our results indicates that the herein presented algorithm is a promising alternative to existing methods. The algorithm proposed in [12] produce almost identical results as the algorithm (9-16) which is not surprising since, as we have demonstrated, for uniformly spaced frequencies the two methods are closely related.

\subsubsection{Validation}

In order to validate the estimates we divided the JPL-data in two sets as described previously. In figure 3 the results of estimating models of order 19-43 is given. It is interesting to notice that the identification error measured on the validation data keeps decreasing which indicates that the JPL-data is fairly noise free and origins from a system of a high order, possibly infinite.

\section{Conclusions}

We have in this paper presented a non-iterative frequency domain state-space identification algorithm. If the frequency data is noise-free and generated by an $n$th order system we show that only $n+2$ equidistant frequency response samples are required to exactly recover the true system. We also demonstrate that the algorithm is related to an existing algorithm [12]. The proposed algorithm is used to identify a state space model from real data originating from a flexible structure 


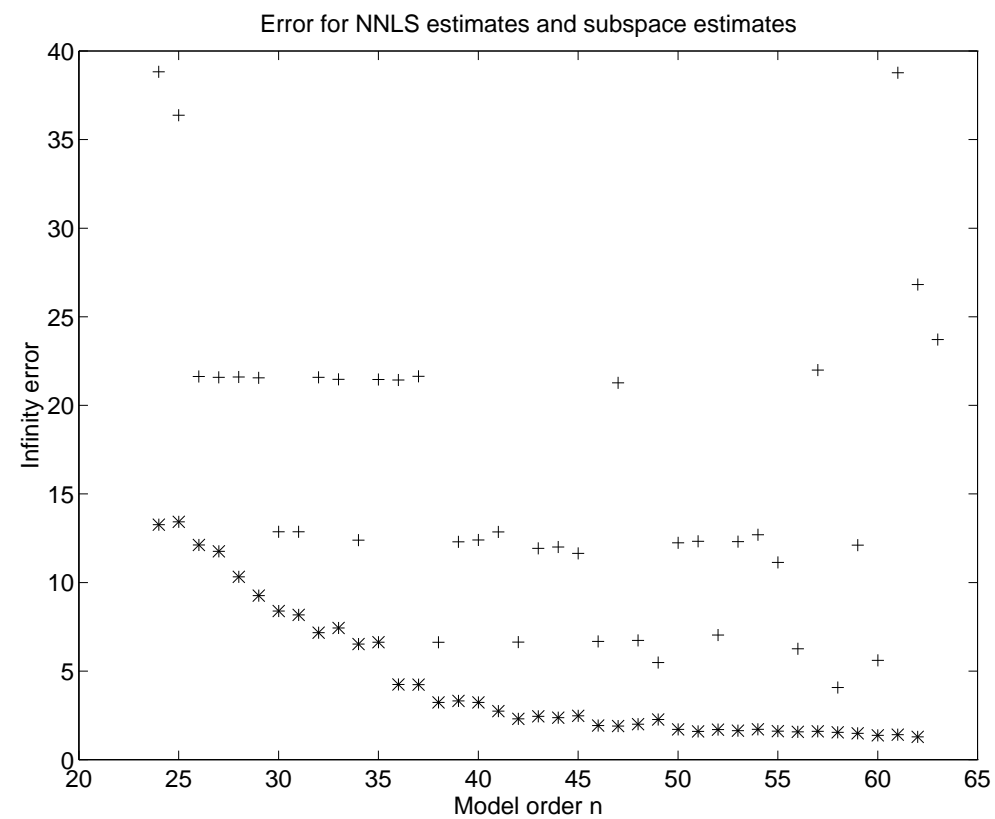

Figure 2: $\|G-\hat{G}\|_{\infty e}$ error for different model orders. "*” Algorithm (9-16), "+" Nonlinear least-squares method.

and a comparison is made with a nonlinear least-square iterative method. The results suggest that the algorithm outperforms the nonlinear-least squares algorithm (invfreqz) and is therefore an appealing alternative to classical iterative methods.

\section{References}

[1] J. L. Adcock. Curve fitter for pole-zero analysis. Hewlett-Packard Journal, pages 33-36, January 1987.

[2] D. S. Bayard. An algorithm for state-space frequency domain identification without windowing distortions. In Proc. of the 31st IEEE Conference on Decision and Control, Tucson, Arizona, pages 1707-1712, December 1992.

[3] R. L. Dailey and M. S. Lukich. Mimo transfer function curve fittting using chebyshev polynomials. In Proc. of the SIAM 35th Anniversary Meeting, Denver, Colorado, 1987.

[4] K. Glover. All optimal hankel-norm approximations of linear multivariable systems and their $L^{\infty}$-error bounds. International Journal of Control, 39(6):1115-1193, 1984.

[5] G. Gu and P. P. Khargonekar. Frequency domain identification of lightly damped systems: The jpl example. In Proc. of the American Control Conference, San Francisco, CA, pages 3052-3056, 1993.

[6] B. L. Ho and R. E. Kalman. Effective construction of linear state-variable models from input/output functions. Regelungstechnik, 14(12):545-592, 1966. 


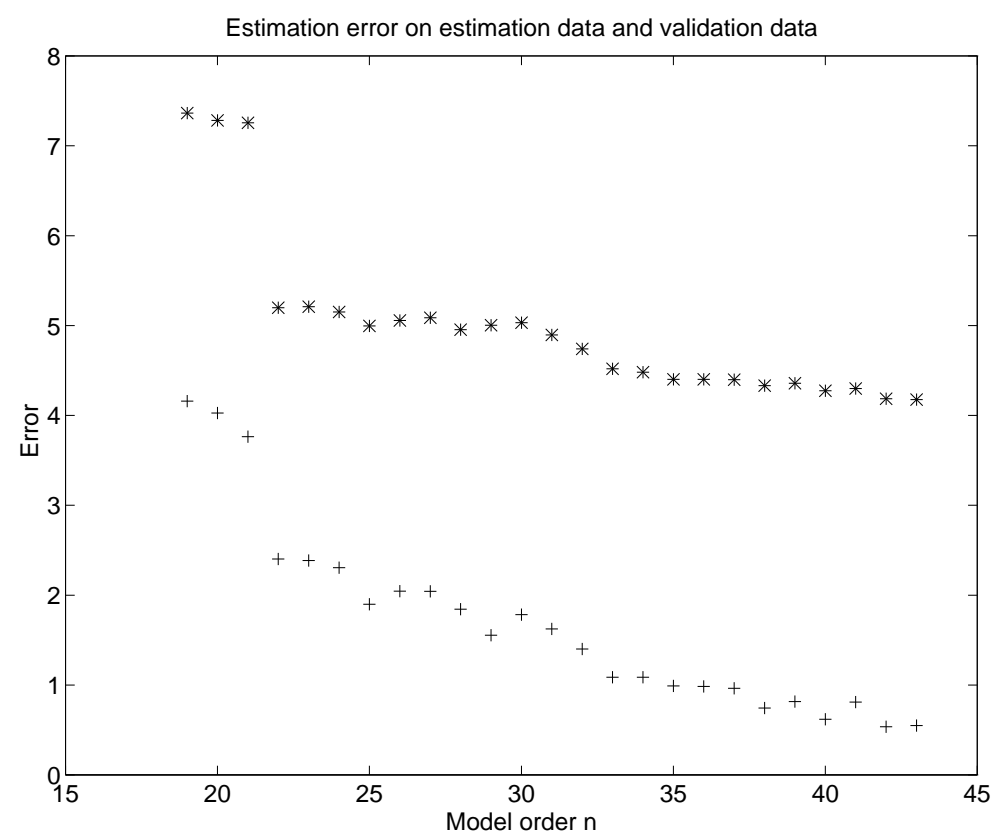

Figure 3: $\|G-\hat{G}\|_{2 e}$ error on estimation data "+" and validation data "*" for different model orders using algorithm (9-16).

[7] J. N. Juang and R. S. Pappa. An eigensystem realization algorithm for modal parameter identification and model reduction. J. of Guidence, Control and Dynamics, 8(5):620-627, 1985 .

[8] J. N. Juang and H. Suzuki. An eigensystem realization algorithm in frequency domain for modal parameter identification. Journal of Vibration, Acoustics, Stress, and Reliability in Design, 110:24-29, January 1988.

[9] S. Y. Kung. A new identification and model reduction algorithm via singular value decomposition. In Proc. of 12th Asilomar Conference on Circuits, Systems and Computers, Pacific Grove, CA, pages 705-714, 1978.

[10] E. C. Levy. Complex curve fitting. IRE trans. on automatic control, AC-4:37-44, May 1959.

[11] J. Little and L. Shure. Signal Processing Toolbox. The Mathworks, Inc., 1988.

[12] K. Liu, R. N. Jacques, and D. W. Miller. Frequency domain structural system identification by observability range space extraction. Technical report, Space Engineering Research Center, MIT Cambridge, MA 02139, September 1993. Submitted for review for 1994 ACC and ASME Journal of Dynamic Systems, Measurment and Control.

[13] K. Liu and R. E. Skelton. Q-markov covariance equivalent realization and its application to flexible structure identification. AIAA Journal of Guidance, Control and Dynamics, 16(2):308319, 1993. 
[14] L. Ljung. System Identification: Theory for the User. Prentice-Hall, Englewood Cliffs, New Jersey, 1987.

[15] T. McKelvey, H. Akçay, and L. Ljung. Efficient construction of transfer functions from frequency response data. Technical report, Report LiTH-ISY-xxxx, Dep. of EE, Linköping University, S-581 83 Linköping, Sweden, 1994.

[16] M. Moonen, B. De Moor, L. Vandenberghe, and J. Vandewalle. On- and off-line identification of linear state-space models. International Journal of Control, 49(1):219-232, 1989.

[17] L. E. Pfeffer. The rpm toolbox: A system for fitting linear models to frequency response data. In Proc. of 1993 MATLAB Conference, Cambridge, MA, October 1993.

[18] C. K. Sanathanan and J. Koerner. Transfer function synthesis as a ratio of two complex polynomials. IEEE Trans. Automatic Control, 8:56-58, January 1963.

[19] P. Stoica, P. Eykhoff, P. Janssen, and T. Söderström. Model structure selection by cross validation. International Journal of Control, 43:1841-1878, 1986.

[20] M. Verhagen. A novel non-iterative mimo state space model identification technique. In Proc. 9th IFAC/IFORS Symp. on Identification and System parameter estimation, Budapest, Hungary, pages 1453-1458, July 1991.

[21] A. H. Whitfield. Asymptotic behaviour of transfer function synthesis methods. Int. Journal of Control, 45(3):1083-1092, 1987. 